\title{
Fatores sociocognitivos e resultados da gestão pessoal da carreira em
}

$$
\text { desempregados portugueses }{ }^{i}
$$

\section{Sociocognitive factors and results of career self-management in unemployed}

\author{
adults \\ Maria do Céu Taveira*, Bárbara Cardoso* Cátia Marques* \\ *Escola de Psicologia, Universidade do Minho
}

\begin{abstract}
Resumo
A gestão pessoal da carreira é um processo continuado de reflexão, definição de objetivos e autorregulação na vida de trabalho. Pretende-se testar um modelo sociocognitivo do constructo, aplicado aos comportamentos de exploração e decisão, em adultos desempregados portugueses ( $N=427$; $n=234,54.8 \%$ mulheres), com idades entre 17 e 65 anos $(M=47.10 ; D P=6.5)$, e residência e inscrição em centros de emprego das diferentes regiões do país. Os resultados de uma análise de caminhos indicam que o modelo testado se ajusta globalmente aos dados e pode constituir uma base teórica útil à investigação e intervenções de carreira com desempregados.

Palabras clave: teoria sociocognitiva, modelo de gestão pessoal da carreira, exploração, tomada de decisão.
\end{abstract}

\begin{abstract}
Career self-management is a continuing process of reflection, goal setting and self-regulation in work and personal life. The aim of this study is to test a sociocognitive model of the construct applied to career exploration and decision-making in Portuguese unemployed adults ( $N=427 ; n=234,54.8 \%$ women $)$, aged 17-65 years old $(M=47.10 ; D P=6.5)$, with residence and enrollment in employment centers of the different regions of the country. The results of a path analysis indicate that the model tested fits the data globally and can provide a useful theoretical basis for research and career interventions with the unemployed.

Keywords: social cognitive theory,

career self-management model, career exploration, career decision-making.
\end{abstract}

Entrar no mundo de trabalho atualmente requer um maior esforço da parte dos indivíduos: exige um autoconhecimento mais profundo e um sentimento generalizado de domínio sobre a própria vida/carreira (e.g., Pinto, Ordonez, \& Taveira, 2015). Por sua vez, a mudança frequente de empregos, a instabilidade e imprevisibilidade dos percursos profissionais e a evolução da natureza do trabalho, nas sociedades contemporâneas, tem vindo a questionar o conceito de carreira tradicional (Briscoe, Hall, \& DeMuth, 2006; Savickas, 2012). Lent e Brown (2013) propõem que se utilize o termo carreira de forma genérica, para abranger o trabalho ou o comportamento ocupacional, independentemente do nível de prestígio da ocupação. Neste sentido, podemos considerar que um construtor civil, tal como um médico, tem uma carreira. Outros autores fazem notar que a carreira passou a ser de natureza multiforme e deve designar o conjunto total de experiências de trabalho e de aprendizagem no decurso da vida (Pinto et al., 2015). Freitas, Ferreira, Santos, \& Fernandes (2014) defendem, por isso, que um maior sentimento de auto-eficácia, maior locus de controlo interno, e bons índices de autoestima, poderão ser sinónimos de um sentimento generalizado de domínio sobre a própria vida/carreira, e ter importantes efeitos na preparação da procura de emprego e na redução de stress em processos de transição na carreira. Vieira, Maia e Coimbra (2007) argumentam, além disso, que no final de um percurso académico, a proximidade da entrada no mercado do trabalho suscita a reflexão acerca da capacidade para conseguir um emprego. Se considerarmos a influência deste sentido de autoeficácia na autorregulação comportamental e na persistência perante obstáculos (Bandura, 2000; Betz \& Voyten, 1997), podemos antecipar que a confiança na capacidade dos indivíduos para lidar com a transição para o mercado de trabalho ou com transições de emprego, pode tornálos mais proactivos, determinados e perseverantes nas suas estratégias de procura de emprego (Lent \& Brown, 2013). Será importante ainda que, perante a natureza mais mutável dos contextos de trabalho, os indivíduos desenvolvam outras competências de adaptabilidade de carreira (e.g., planeamento, exploração, tomada de decisão) e competências de aprendizagem, que lhes permitam lidar eficazmente e de um modo continuado, com problemas e exigências não previstas (Savickas, 2005).

Neste sentido, a gestão pessoal de carreira é um conceito que pode responder a estes desafios, já que designa um processo continuado de reflexão sobre si próprio/a e sobre a sua história de vida, e a capacidade de exploração e decisão sobre diferentes situações do quotidiano, com um foco nas questões do trabalho (Savickas, 2012). Neste âmbito, torna-se fundamental que cada individuo entenda e aja sobre os fatores proximais que podem influenciar a gestão da sua carreira profissional, bem como sobre aqueles que estão fora do seu alcance, e sobre os quais poderá ter uma influencia mais indireta (e.g., Lent \& Brown, 2013; Loureiro, 2012). Nestes moldes, pensar sobre a carreira e tomar decisões, 
não é um exercício que se faz apenas quando se está já no mercado de trabalho. Todas as decisões tomadas ao longo do percurso académico e profissional são relevantes e podem ajudar a desenvolver um projeto de vida que vá não só ao encontro de expectativas e objetivos sociais, como também, dos objetivos pessoais.

As teorias sobre o processo de gestão pessoal da carreira (e.g., Greenhaus \& Callanan, 1994; Greenhaus, Callanan, \& Godshalk 2010; King, 2004) descrevem-no como um processo cíclico, através do qual os indivíduos exploram a sua carreira e recolhem informações sobre interesses e competências, o que, por sua vez, favorece o recurso a estratégias de carreira que aumentam a probabilidade de alcance de objetivos pessoais de carreira. Neste âmbito, Lent e Brown (2013) apresentam um modelo sociocognitivo explicativo da adaptação ao desemprego e dos comportamentos de gestão de carreira na transição para o emprego ou para um novo emprego, durante a vida adulta. De acordo com este modelo, são exemplos de comportamentos adaptativos a desenvolver e a exibir quando os indivíduos estão a tentar estabelecerse no mercado de trabalho: continuar a explorar a carreira, procurar um emprego, lidar com eventos negativos, como a perda de emprego, rever ou estabilizar objetivos e planos vocacionais, refinar competências interpessoais e politicas, e envolver-se em comportamentos de cidadania (Lent \& Brown, 2013, p.560).

Esta teoria constitui um quadro concetual importante para desenhar e desenvolver estudos sobre a gestão pessoal da carreira, nomeadamente com desempregados, no sentido de compreender melhor que condições sociais e cognitivas podem caracterizar e ajudar a explicar o processo, neste grupo específico, dada a importância da transição para o emprego ou para um novo emprego, na vida adulta. O modelo proposto por Lent e Brown (2013) foca como variáveis exógenas, os inputs da pessoa (e.g., idade, sexo, etnia), do contexto (e.g., a educação), as experiências de aprendizagens (e.g., fontes de autoeficácia), a personalidade, e influências do contexto (apoios ou barreiras) próximas dos comportamentos adaptativos; como variáveis endógenas, inclui as expectativas de resultados, a autoeficácia, os objetivos de carreira valorizados, e os comportamentos adaptativos propriamente ditos (e.g., exploração, tomada de decisão); e, como efeitos deste processo, resultados de carreira pretendidos (e.g., mais certeza vocacional, menos stress, obtenção de emprego).

Lent, Ezeofor, Morrison, Penn, \& Ireland (2016) testaram o seu modelo pela primeira vez com estudantes universitários finalistas de Psicologia, e verificaram que (a) a conscienciosidade, e o suporte social percebido na exploração e decisão, se relacionavam significativamente com a autoeficácia percebida na exploração e decisão; (b) o apoio percebido e a autoeficácia em conjunto prediziam as expectativas de resultado em relação à exploração e tomada de decisão; (c) o apoio percebido, a autoeficácia e as expectativas de resultado prediziam as intenções ou objetivos de exploração e de tomada de decisão. Além disso, a autoeficácia prediz negativamente a ansiedade face à exploração e à tomada de decisão e positivamente a certeza vocacional (Lent et al., 2016, p.54). Os resultados deste estudo apoiam de modo significativo as hipóteses do modelo de gestão pessoal da carreira sociocognitivo em universitários.

Este estudo destina-se a testar o referido modelo em adultos desempregados portugueses, para explicar as intenções e comportamentos adaptativos de exploração e decisão deste grupo da população. Para o efeito, foram definidas as seguintes hipóteses de investigação:

H1. A personalidade afeta diretamente as intenções e comportamentos de exploração e decisão e, indiretamente, através da autoeficácia;

H2. Os apoios percebidos afetam diretamente as intenções e comportamentos de exploração e decisão e, indiretamente, através da autoeficácia e expectativas de resultado;

H3. A autoeficácia contribui diretamente para as intenções e comportamentos de exploração e decisão e, indiretamente, e através das expectativas de resultados;

H4. As expectativas de resultado afetam direta e positivamente as intenções e comportamentos de exploração e decisão;

H5. As intenções e comportamentos de exploração vocacional e de tomada de decisão afetam positivamente a certeza vocacional e negativamente a ansiedade face à exploração e tomada de decisão.

\section{Método}

\section{Participantes}

A amostra foi intencional, não probabilística. Inclui um total de 427 adultos desempregados de ambos os sexos, 234 mulheres $(54.80 \%)$ e 193 homens $(45.20 \%)$, com uma média de idades de 47.10 anos $(D P=6.5)$, inscritos em centros de emprego das diferentes regiões do país, com um diploma de estudos do ensino superior (222, $52 \%$ mestres; 136, 31.9\% licenciados; 3, 7\% doutorados), do ensino secundário $(54 ; 12.6 \%)$, ou do ensino básico $(12,2.8 \%)$. O recrutamento dos participantes efetuou-se através do recurso ao Instituto de Emprego e Formação Profissional (IEFP), que disponibilizou uma base de dados constituída por 28061 desempregados de todo o país que consentiram a divulgação dos seus contactos. Desse total, foram retirados à sorte, 4000 endereços eletrónicos, para onde foi enviada uma mensagem eletrónica com a apresentação do projeto, consentimento informado e protocolo de avaliação. Daquelas, cerca de 100 mensagens foram devolvidas, devido a endereço inválido. Das restantes 3900 mensagens, obteve-se um total de 527 respostas. Destas, 427 foram consideradas válidas, por incluírem respostas a todas as medidas.

\section{Instrumentos}

A recolha de dados recorreu a um protocolo digital na plataforma SurveyMonkey ${ }^{\circledR}$, com diferentes medidas:

Questionário de Dados Sociodemográficos. Elaborado para o efeito do estudo, com oito questões, para recolha de dados gerais (e.g., sexo), escolares (e.g., habilitações) e profissionais (e.g., trabalhos executados).

NEO-FFI (Five Factor Model: FFM; McCrae \& Costa, 2004). Foi utilizada a versão portuguesa breve do NEO-FFI (Magalhães et al., 2014), com 60 itens (12 por 
dimensão), respondidos numa escala de cinco pontos ( $0=$ discordo fortemente; $4=$ concordo fortemente) que avaliam a abertura à experiência (e.g., "Fico admirado(a) com os modelos que encontro na arte e na natureza"), o neuroticismo (e.g., "Muitas vezes sinto-me tenso(a) ou enervado(a)"), a conscienciosidade (e.g., "Mantenho as minhas coisas limpas e em ordem"), a amabilidade (e.g., "Tenho de ser delicado(a) com todas as pessoas que encontro") e a extroversão (e.g., "Rio facilmente"). Magalhães et al. (2014) registaram valores de consistência interna satisfatórios de conscienciosidade $(\alpha=.81)$, neuroticismo $(\alpha=.81)$, extroversão $(\alpha=.75)$, amabilidade $(\alpha=.72)$ e abertura à experiência $(\alpha=.71)$, escala total $(\alpha=.82)$. Neste estudo, os coeficientes de alfa variaram de satisfatórios a aceitáveis, com $\alpha=.83$ para conscienciosidade, $\alpha=.73$ para neuroticismo, $\alpha=.77$ para extroversão, $\alpha=.60$ para amabilidade e $\alpha=.67$ para abertura à experiência. Influence of Others on Academic and Career Decision Making Scale (IOACDS; Nauta \& Kokaly, 2001). Foi utilizada a versão portuguesa, de Freitas e col. (2014), que avalia o suporte social na carreira constituída por oito itens (e.g., "Tenho alguém com quem posso contar se precisar de apoio numa tomada de decisão escolar e profissional"), respondidos numa escala de cinco pontos, ( $1=$ discordo fortemente $5=$ concordo fortemente), e com níveis satisfatórios de precisão $(\alpha=.77)$ no estudo original (Freitas et al., 2014) e neste estudo $(\alpha=.83)$.

Career-Exploration and Decision Self-Efficacy Scale (CEDSE, Lent et al., 2016). Avalia a autoeficácia na exploração (8 itens; e.g., "Identificar carreiras que melhor fazem uso das suas capacidades") e tomada de decisão (4 itens; e.g., "Lidar com a deceção se a primeira escolha não funcionar"), com resposta numa escala de cinco pontos (1=nada confiante; 5=completamente confiante), e valores de fiabilidade satisfatórios $(\alpha=.94$ e $\alpha=.96)$ no estudo original e neste estudo ( $\alpha=.95$ e $\alpha=$ .90). Dado não existir versão portuguesa da escala, a estrutura fatorial da CEDSE foi validada através de análise fatorial confirmatória (AFC) com recurso ao software AMOS (v.24, SPSS Inc, Chicago, IL), registando-se um ajustamento global razoável do modelo bifatorial, com valores de teste do $\chi^{2}$ de 513.82 (gl= 53, $p<.001)$, de RMR $=.056$, de Comparative Fit Index $(\mathrm{CFI})$ de .882, de Root Mean Square Error of Approximation (RMSEA) de .152 e de Goodness of Fit Index (GFI) de .788. Este modelo foi escolhido, tendo apresentado melhor ajuste aos dados, quando comparado com um modelo unifatorial, alternativo (Cardoso, 2017).

Career Outcome Expectations and Exploratory Intentions Scale (COEEIS; Betz \& Voyten, 1997). Avalia as expectativas de resultados e intenções de exploração (5 itens; e.g., "Tenciono despender mais tempo do que já despendi a explorar oportunidades de carreira") e de tomada de decisão (4 itens; e.g., "Se eu conhecer os meus interesses e capacidades, estou capaz de escolher uma boa carreira"), duas subescalas respondidas numa escala de cinco pontos, $(1=$ discordo fortemente; $5=$ concordo fortemente), com índices de precisão satisfatórios $(\alpha=.79$ e $\alpha=.73)$, incluindo neste estudo ( $\alpha=.85$ e $\alpha=.79)$. Dado não existir versão portuguesa da escala, a estrutura fatorial da COEEIS foi validada através de uma AFC, obtendo-se um ajustamento global do modelo original, com valores de teste do $\chi^{2}$ de 132.274 (gl= 26, $\left.p<.001\right)$, de $\mathrm{RMR}=.054$, de CFI de .921, de RMSEA de .104 e de GFI de 925 (Cardoso, 2017).

Career Exploration Survey (CES; Stumpf, Colarelli \& Hartman, 1983). Utilizou-se a versão portuguesa (Taveira, 1997), e apenas quatro das 12 subescalas que compõem a medida: Exploração do meio (5 itens; e.g., "Nos últimos três meses, tentei obter informações sobre algumas profissões e empregos"); Exploração de si próprio ( 5 itens; e.g., "Nos últimos três meses, pensei na minha vida escolar/académica passada"); Stress na exploração (4 itens; e.g., "Ir a entrevistas em empresas específicas, na minha área profissional preferida"); e o Stress na decisão (5 itens; e.g., "Decidir-me por uma profissão"), respondidas numa escala com cinco pontos no caso das primeiras duas ( $1=$ muita $5=$ muito pouca $)$ e sete pontos nas restantes (1=tensão mínima; 7= tensão máxima). A CES registou valores aceitáveis a satisfatórios de fiabilidade $(.63<\alpha<.86)$ no estudo original, e satisfatórios, neste estudo $(.80<\alpha<.93)$.

Escala de Certeza Vocacional (ECV; Santos, 2007). A ECV é constituída por quatro itens (e.g., "Já escolhi uma determinada opção profissional da qual não tenciono afastar-me"), respondidos numa escala de seis pontos ( $1=$ discordo inteiramente; $6=$ concordo inteiramente $)$, com níveis de precisão satisfatórios, no estudo original ( $\alpha$ $=.85)$ e neste estudo $(\alpha=.89)$.

\section{Procedimentos}

A mensagem de apresentação do estudo e ligação ao consentimento informado e protocolo de avaliação foram enviados por correio eletrónico a cada participante. Todos preencheram o protocolo de avaliação de forma voluntária, sem recompensas materiais, garantindo-se a confidencialidade no tratamento e divulgação dos dados e resultados.

Foram realizadas análises descritivas, correlação de Pearson, confiabilidade de escalas (alfa de Cronbach), fatorial confirmatória, regressão linear múltipla e de caminhos (path analysis) com recurso ao software AMOS (v.22, SPSS, IBM Company, Chicago, IL).

\section{Resultados}

A conscienciosidade apresentou a média $(M=49.4)$ mais elevada, e o total da autoeficácia na exploração e tomada de decisão o desvio-padrão mais elevado $(D P=$ 10.7). Quanto às correlações, registou-se um valor mais elevado entre o total da escala da autoeficácia e a autoeficácia na exploração vocacional $(r=.95)$, e entre as intenções de exploração e decisão e as expectativas de resultado relacionadas $(r=.90)$. A correlação mais baixa verificou-se entre o neuroticismo e a extroversão ( $r=$ .49). A consistência interna das escalas variou de aceitável a satisfatória $(60<\alpha<96)$.

As medidas da personalidade e do apoio social foram testadas separadamente como preditores das variáveis sociocognitivas e estas como preditores das intenções e comportamentos de exploração e decisão vocacional. Testou-se ainda o papel destas últimas no stress e certeza vocacional. Os resultados indicaram que a conscienciosidade é a dimensão da personalidade que 
afeta mais dimensões vocacionais, quando comparada com as restantes, tendo sido escolhida para o teste final do modelo. Na análise de caminhos, a significância dos coeficientes de regressão foi avaliada após a estimação dos parâmetros, pelo método da máxima verosimilhança. O cumprimento dos pressupostos inerentes a esta análise foi previamente verificado (Marôco, 2014). Na tabela 1, apresentam-se os resultados da análise do ajustamento do modelo original aos dados, com a conscienciosidade como variável exógena; e o ajustamento do modelo especificado, resultante da aplicação dos índices de modificação sugeridos (e.g., distúrbios correlacionados). A análise foi realizada sem consideração dos casos outliers $(n=4)$, com um total de 381 participantes $(216$, $56,7 \%$ mulheres), dos 17 aos 65 anos $(M=45 ; D P=6.2)$.

Tabela 1.

Ajustamento aos dados dos modelos de gestão pessoal de carreira aplicados à exploração e tomada de decisão

\begin{tabular}{lcccccc}
\hline & $X^{2}$ & df & $p$ & RMSEA & CFI & GFI \\
\hline Inicial & 418.04 & 6 & .000 & .425 & .169 & .783 \\
Final & 110.43 & 9 & .000 & .172 & .796 & .929 \\
\hline
\end{tabular}

Nota: Inicial é o modelo de gestão pessoal de carreira original com a conscienciosidade como fator de personalidade. Final é o modelo inicial especificado.

A análise dos índices de modificação sugeriu uma relação entre um distúrbio do suporte social (e6) com a auto-eficácia; um distúrbio do suporte social (e8) com o stress decisional; e um distúrbio do suporte social (e10) com a conscienciosidade. Consideradas estas modificações, o modelo final regista um ajustamento razoável aos dados, com uma melhoria significativa em todos os índices (Figura 1).

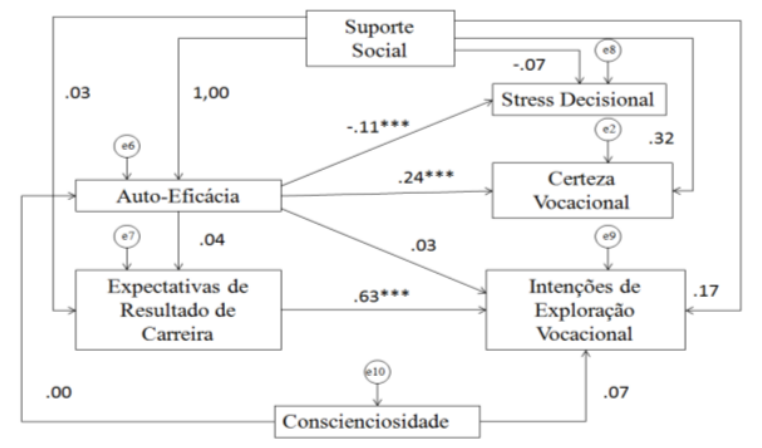

Figura 1. Modelo final: Diagrama da path analysis $(\mathrm{n}=381) . * * * \mathrm{p}<.001$

A conscienciosidade é um preditor estatisticamente significativo da auto-eficácia $\mathrm{R}^{2}=.12, p<.001 \mathrm{e}$ das intenções de exploração vocacional $\mathrm{R}^{2}=.13, p<.001$. Os apoios percebidos afetam diretamente as intenções de exploração e decisão, e indiretamente, através da autoeficácia e expectativas de resultado. O modelo apresentou caminhos significativos que envolvem o total da autoeficácia na exploração e decisão $\left(\mathrm{R}^{2}=.08, p<\right.$ $.001)$, as expectativas de resultado com a exploração e decisão e a certeza vocacional $\left(\mathrm{R}^{2}=.05, p<.001\right)$, as intenções de exploração vocacional e o stress na decisão
$\left(\mathrm{R}^{2}=.23, p<.01 ; \mathrm{R}^{2}=.03, p<.01\right.$, respetivamente $(\mathrm{H} 1 \mathrm{e}$ H2). A autoeficácia é um preditor estatisticamente significativo das expectativas de resultado $\mathrm{R}^{2}=.05, p<$ .001 , intenções de exploração $\mathrm{R}^{2}=.07, p<.001$, certeza vocacional $\mathrm{R}^{2}=.27, p<.001$ e stress na decisão $\mathrm{R}^{2}=.05$, $p<.001(\mathrm{H} 3)$. As expectativas de resultado afetam positivamente as intenções de exploração e decisão $\mathrm{R}^{2}=$ $.38, p<.001(\mathrm{H} 4)$. A Hipótese 5 não se verificou, dado o caminho predefinido não ser significativo.

\section{Discussão e conclusão}

O objetivo principal do presente estudo foi testar o modelo sociocognitivo de gestão pessoal da carreira de Lent e Brown (2013) aplicado à exploração e tomada de decisão vocacional em adultos desempregados portugueses. Neste estudo, a conscienciosidade foi a dimensão da personalidade que apresentou relações significativas previstas com a auto-eficácia na exploração e decisão vocacional, as quais estabelecem associações significativas com as intenções em explorar e tomar decisões de carreira (H1). A conscienciosidade favorece a autoeficácia em tarefas de planeamento de carreira, já que envolve a capacidade de organização e persistência, permitindo sustentar o sentido de competência em tarefas vocacionais, como a exploração e a decisão (Lent et al., 2016). O suporte social afecta também positivamente a autoeficácia e, negativamente, a ansiedade decisional (H2). Os desempregados que usufruem de relações com pessoas em quem confiam e que ajudam na exploração de carreira e escolhas vocacionais, sentem-se mais competentes nestas tarefas de carreira e antecipam menor stress em relação à tomada de decisão vocacional. $\mathrm{O}$ suporte social é um preditor significativo das intenções de exploração, das expectativas de resultado com a exploração e decisão, e da certeza vocacional. Este resultado evidencia o papel importante do suporte social na explicação de crenças e comportamentos chave para lidar com transições de carreira, o que é consistente com evidência anterior (e.g., Lent et al., 2016). Quanto à terceira hipótese, verificou-se que a auto-eficácia é um preditor estatisticamente significativo das intenções de exploração vocacional, tendo um efeito indireto nestas, através das expectativas de resultados, e afetando direta e positivamente, também, a certeza vocacional e, negativamente, a ansiedade face à tomada de decisão. Indivíduos desempregados com autoeficácia na tomada de decisão de carreira, sentem-se mais confiantes, e evidenciam menos ansiedade face a decisões de carreira e maior certeza vocacional. Neste sentido, a auto-eficácia assume uma grande importância no que toca às questões de gestão pessoal de carreira, envolvendo crenças sobre a capacidade para gerir tarefas de carreira especificas e adaptativas. Estes resultados reforçam evidência sobre o valor explicativo da auto-eficácia no âmbito dos comportamentos e processos de carreira (Vieira, Maia \& Coimbra, 2007). As expectativas de resultado dos desempregados estudados predizem positivamente as suas intenções de exploração vocacional, o que nos conduz à ideia de que um individuo desempregado com expectativas de resultado de carreira elevadas, tende a querer explorar e a envolver-se na tomada decisão, 
aspetos importantes do processo de gestão pessoal da carreira, em especial, em fases de transição vocacional (e.g., Freitas et al., 2014). As intenções de exploração e decisão juntamente com os comportamentos de exploração e decisão, não surgiram como preditores significativos dos resultados de carreira (certeza ou ansiedade) (H5), sendo estes preditos, antes, pelo suporte social e autoeficácia. Este resultado remete para a importância de analisar mais aprofundadamente, em futuros estudos, a natureza dos comportamentos de gestão pessoal da carreira e as suas consequências, tendo por base a teoria sobre a gestão de carreiras (e.g., Greenhaus et al., 2010).

O modelo sociocognitivo de gestão pessoal da carreira evidenciou ter um poder explicativo importante em crenças e resultados de carreira desejados, na população de desempregados, como as intenções de explorar novas oportunidades de carreira, certeza em relação ao papel de trabalhador e ansiedade não debilitante. Deste modo, deve-se procurar auxiliar as pessoas no sentido de criar $\mathrm{e}$ valorizar intervenções de carreira existentes e direcionálas para a promoção de atitudes de planeamento, angariação de apoios, e crenças positivas em relação ao exercício intencional e sistemático da exploração e da tomada de decisão.

\section{Referências}

Bandura, A. (2000). Self-efficacy: The foundation of agency. In W. J. Perrig \& A. Grob (Eds.), Control of human behaviour, mental processes and consciousness (pp. 17-33) Mahwak, NJ: Erlbaum.

Betz, N. E., \& Voyten, K. K. (1997). Efficacy and outcome expectations influence career exploration and decidedness. The Career Development Quarterly, 46, 179-189. doi: 10.1002/j.2161-0045.1997.tb01004.x

Briscoe, J. P., Hall, D. T., \& DeMuth, R. L. (2006). Protean and boundaryless careers: An empirical exploration. Journal of Vocational Behavior, 69, 30 47. doi: 10.1016/j.jvb.2005.09.003

Cardoso, B. E.C. (2017). Fatores sociocognitivos da gestão pessoal da carreira em desempregados Portugueses (Tese de mestrado integrado não publicada). Universidade do Minho, Braga.

Freitas, R. A., Ferreira, J. A., Santos, E., \& Fernandes, D. (2014). Reinserção profissional de desempregados: Procura ativa e padrões motivacionais. Área temática 7-formação e transição para o mundo de trabalho, 3388 .

Greenhaus, J. H., \& Callanan, G. A. (1994). Career management $\left(2^{\text {nd }}\right.$ ed.). Fort Worth, TX: The Dryden Press.

Greenhaus J. H., Callanan, G.A., \& Godshalk, V.M. (2010). Career Management ( $4^{\text {th }}$ ed.). Thousand Oaks, CA: Sage.

King, Z. (2004). Career self-management: Its nature, causes and consequences. Journal of Vocational Behavior, 65, 112-133. doi.org/10.1016/S00018791(03)00052-6
Lent, R. W., \& Brown, S. D. (2013). Social cognitive model of career self-management: Toward a unifying view of adaptive career behavior across the life span. Journal of Counseling Psychology, 60, 557-56. doi: 10.1037/a0033446

Lent, R. W., Ezeofor, I., Morrison, M. A., Penn, L. T., \& Ireland, G.W. (2016). Applying social cognitive model of career self-management to career exploration and decision-making. Journal of Vocational Behavior, 93, 47-57. doi: 10.1016/j.jvb.2015.12.007

Loureiro, M.N. (2012). Intervenção vocacional no ensino superior: Estudo de avaliação da eficácia do Seminário de Gestão Pessoal de Carreira-Versão A (Tese de doutoramento não publicada). Universidade do Minho, Braga.

Magalhães, E., Salgueira, A., Gonzalez, A. J., Costa, J. J., Costa, M. J., \& Costa, P. (2014). A versão portuguesa do NEO-FFI: Caracterização em função da idade, género e escolaridade. Revista Psicologia, 28(2), 1-10.

Marôco, J. (2014). Análise estatística com o SPSS Statistics (6 ${ }^{\mathrm{a}}$ ed.). Lisboa: Report Number.

McCrae R. R., \& Costa P. T. (2004). A contemplated revision of the NEO five-factor inventory. Personality and Individual Differences, 36, 587596. doi:10.1016/S0191-8869(03)00118-1

Nauta, M. M., \& Kokaly, M. L. (2001). Assessing role model influences on students' academic and vocational decision. Journal of Career Assessment, 9, 81-99. doi: 10.1177/106907270100900106

Pinto, J. C., Ordónez, J. L., \& Taveira, M.C. (2015). Cómo orientar la gestión de la carrera profesional. Barcelona: Editorial UOC.

Santos, P. J. (2007). Dificuldades de escolha vocacional. Coimbra: Almedina.

Savickas, M. L. (2005). The theory and practice of career construction. In S. D. Brown \& R. W. Lent (Eds.), Career development and counseling: Putting theory and research to work (pp. 42-70). Hoboken, NJ: John Wiley \& Sons.

Savickas, M. L. (2012). Life design: A paradigm for career intervention in the 21 st century. Journal of Counseling \& Development, 90, 13-19. doi: 10.1111/j.1556-6676.2012.00002.x

Stumpf, S. A., Colarelli, S. M., \& Hartman, K. (1983). Development of the career exploration survey (CES). Journal of Vocational Behavior, 22, 191-226. doi: 10.1016/0001-8791(83)90028-3

Taveira, M. C. (1997). Exploração e desenvolvimento vocacional de jovens: Estudo sobre as relações entre a exploração, a identidade e a indecisão vocacional (Dissertação de Doutoramento não publicada) Universidade do Minho, Braga.

Vieira, D., Maia, J., \& Coimbra, J.L. (2007). Do ensino superior para o trabalho: Análise factorial confirmatória da escola de auto-eficácia na transição para o trabalho (AETT). Avaliação Psicológica, 6(1), 3-12.

\footnotetext{
i Uma primeira apresentação deste trabalho foi realizada em 2017, na Universidade do Minho, pela segunda autora, numa tese de mestrado integrado em Psicologia.
} 